\title{
Platelet Indices and Erythrocyte Parameters in Apparently Healthy Police Dogs in Khartoum State, Sudan
}

\author{
${ }^{1 *}$ Abdullah, A. A., ${ }^{2}$ Musa. A. and ${ }^{3}$ Omer, S. A.
}

\author{
${ }^{1}$ Department of Biomedical Sciences, Faculty of Veterinary Science, University of Gadarif, Gadarif, Sudan \\ ${ }^{2}$ Department of Veterinary Surgery, Faculty of Veterinary Medicine, AL-Salam University, Sudan \\ ${ }^{3}$ Department of Biomedical Sciences, College of Veterinary Medicine, Sudan University of Sciences and Technology, Khartoum, Sudan
}

*Author for Correspondence: bahlol32029@gmail.com

\begin{abstract}
The normal levels of platelets and erythrocytes parameters together with the effect of breed and sex on them in healthy police dogs were determined. Forty-six dogs were used; 20 Labrador Retriever and 26 German Shepherd dogs comprising 21 males and 25 females. The dogs were 20-83 months old. Five ml blood samples were collected from the cephalic vein; and analyzed using BK6100 Auto Hematology Analyzer. German Shepherd dogs showed significantly $(\mathrm{p} \leq 0.05)$ higher values than Labrador Retriever dogs for Mean platelet volume (MPV) (10.43 \pm 0.71 vs. $9.9 \pm 0.73$ FL), Red blood cells count (RBCs) $\left(6.75 \pm 0.26\right.$ vs. $\left.6.51 \pm 0.27 \times 10^{12} / \mathrm{L}\right)$, and Hemoglobin concentration $(\mathrm{HGB})(180.81 \pm 9.53 \mathrm{vs} .172 .4 \pm 11.98 \mathrm{~g} / \mathrm{L})$, respectively. Highly positive significant correlations $(\mathrm{p} \leq 0.01)$ were found between Platelet count (PLT) and Plateletcrit (PCT) in males, females, and all dogs, and between MPV and Platelets distribution width (PDW) in all dogs. A highly positive significant correlation $(\mathrm{p} \leq 0.01)$ was found between MPV and PCT in all dogs. A highly negative significant correlation $(\mathrm{p} \leq$ 0.01) was found between PDW and RBCs and Red cell blood distribution width- index (RDW-CV) in males and all dogs. Also, a highly positive significant $(\mathrm{p} \leq 0.01)$ correlation was found between PDW and Mean corpuscular volume (MCV) in all dogs. A highly significant negative correlation $(\mathrm{p} \leq 0.01)$ was found between MPV and RBCs in males. A highly negative significant correlation $(\mathrm{p} \leq 0.01)$ was found between MPV and RDW (CV) in males. However, highly positive significant correlations $(\mathrm{p} \leq 0.01)$ were found between MPV and Hematocrit $(\mathrm{HCT})$ in females. It was concluded that breed of dogs may influence platelets and erythrocyte parameters and this should be considered in clinical interpretations.
\end{abstract}

Keywords: Labrador retriever; Erythrocyte parameters; German shepherd; Platelet parameters

\section{INTRODUCTION}

Platelets and Red Blood Cells (RBCs), both of them develop from a common progenitor cell (MEP), and in the peripheral blood, both of them are in anucleated form, but they have an immature peripheral blood-stage called reticulated platelets for platelets and reticulocyte for RBCs (Weiss et al., 2011). Also, erythropoietin, thrombopoietin and a set of cytokine growth factors (IL2, IL4, IL7, etc.) has a significant effect on both the series of cells that gives a special relationship between them (Weiss et al., 2011). Both of them concurrently involved in many physiological rhythms and pathological states as diagnosis and prognosis index (Weiss et al., 2011). Platelet indices and Erythrocyte parameters are biomarkers of platelet activation and RBCs activation respectively. Platelet indices including Mean Platelet Volume (MPV), Plateletcrit (PCT), and Platelet Distribution Width (PDW). Erythrocyte parameters include Red Blood Cell count (RBCs), Hematocrit (HCT), Hemoglobin (Hb), Mean Corpuscular Volume (MCV), Mean Corpuscular
Hemoglobin (MCH), Mean Corpuscular Hemoglobin Concentration (MCHC), and Red Cell Blood Cell Distribution Width- index (RDW- CV). These parameters are available on many automated cell counters (Weiss et al., 2011). Platelets indices and Erythrocyte parameters are commonly used to diagnose the thrombocytopenia, anemia, and other disease conditions in dogs (Bommer et al., 2008; Fabisiak et al., 2010; Temizel et al.,2011; Vojta et al.,2012; Schwartz et al., 2014; Żmigrodzka et al., 2014; Reddy et al., 2015). Breed, sex, and some environmental factors affect the hematological profiles of clinically healthy dogs (Çayir and Kozat, 2016; Schneider and Mischke, 2016; Suljević et $a l ., 2016)$. Many studies have reported the normal values of the hematological profile of dogs all over the world in cold and Tropical climates (Adebiyi et al.,2014; Adekola et al., 2015; Çayir and Kozat, 2016; Schneider and Mischke, 2016; Suljević et al., 2016). Recently, in Sudan, the physiological, diagnostic, and prognostic relevance of platelet indices and Erythrocyte parameters are receiving increasing attention in the field of veterinary medicine (Nidaa et al., 2017). So far, 
there was only one study that has been done to find the reference values of platelet indices and Erythrocyte parameters for German shepherd dogs in Sudan and then to assess the effects of gender on them (Nidaa et al., 2017). Therefore, this study was undertaken to expand our knowledge of the normal ranges of platelet parameters and erythrocyte parameters in clinically healthy dogs from different breeds, and the possible breed and sex effect on them under Sudan conditions. Such information is an important pre-requisite for studies on the usefulness of these parameters in the prognosis, diagnosis, and clinical interpretations of diseases, particularly diseases associated with changes in the blood vessels and bone marrow.

\section{MATERIALS AND METHODS}

\section{Study Design and Animals}

This was a cross sectional study conducted in 2017 in Khartoum State. The dogs belong to the Police Directorate for Dogs at Khartoum State, Burry and they were selected based on their medical history and normal health parameters. Forty-six healthy police dogs, 20 Labrador Retriever, and 26 German Shepherd dogs, comprising 21 males (8 Labrador Retriever and 13 German Shepherd) and 25 females (12 Labrador Retriever and 13 German Shepherd) 20-83 months old. The dogs were individually housed in kennels and fed on an imported dogs' food (Best Signor - Netherlands), and from time to time they were offered other meal consisting of meat soup and noodles, with free access to water.

\section{Blood Collection and Analysis}

Blood samples $(5 \mathrm{ml})$ were collected from the cephalic vein, while the animals were manually restrained; the blood was transferred into a tube with an anticoagulant (EDTA-K ${ }_{3}$ ). Each tube gently inverted 3-5 times to ensure mixture of the sample with the anticoagulant. The samples were immediately transported to the laboratory in an icebox. Using BK6100 Auto Hematology Analyzer- (Biobase, China), the samples analyzed to determine the following parameters: Platelet count (PLT), mean platelet volume (MPV) Plateletcrit (PCT), Platelets distribution width (PDW), Red Blood Cell count (RBCs), Hematocrit (HCT), hemoglobin $(\mathrm{Hb})$, mean corpuscular volume (MCV), Mean Corpuscular Hemoglobin (MCH), Mean Corpuscular Hemo globin Concentration (MCHC) and red cell blood cell distribution width- index (RDW- CV). Quality control of platelets was done using blood smear which was stained with Leishman's stain, A microscope (Olympus, Japan) was used to determine platelet clumps in the dog's blood smear. Samples with large number of clumps were rejected (Weiss et al.,2011).

\section{Statistical Analyses}

All data were sorted and recorded in Microsoft Excel version 2016, cleaned, and then transferred to IBM SPSS version 23.0 for analysis. The data were presented as means \pm standard deviation. Confidence Interval values of the data were also presented. The normality distribution tests were performed using the Kolmogorov-Smirnov test and finally the data were found to be normally distributed. The mean differences of platelets indices and erythrocyte parameters between the study groups were assessed using independent t-test. Pearson correlation were used to find the association among platelet indices and between the platelet indices and erythrocyte parameters. $P$ value of $p<0.05$ was considered significant.

\section{RESULTS}

\section{Platelets Count, Indices and Erythrocyte Parameters}

The mean values, and confidence interval for PLT, MPV, PCT, PDW, RBCs, HCT, Hb, MCV, MCH, MCHC, and RDW- CV for all dogs are presented in Table 1. The mean differences of platelets indices and erythrocyte parameters values in Labrador Retriever and German Shepherd dogs is presented in Table 2. MPV, RBCs, and $\mathrm{Hb}$ were significantly higher $(\mathrm{p} \leq 0.05)$ in the German Shepherd than in the Labrador Retriever. PLT, and PCT, PDW, HCT, $\mathrm{MCV}, \mathrm{MCH}, \mathrm{MCHC}$, and RDW-CV were not significantly different between the two breeds $(\mathrm{p}>0.05)$

Sex-related differences in dogs' platelet indices and erythrocyte parameters are presented in Table 3. PLT, MPV, PCT, PDW, RBCs, HCT, Hb, MCV, MCH, MCHC, and RDW- CV Did not show any significant variation with the $\operatorname{sex}(\mathrm{p}>0.05)$.

\section{Correlation Analysis for Relevant Hematological Variables}

In this study, the correlation between platelet count $\left(\mathrm{PLT}\left(\mathrm{x} 10^{9} / \mathrm{L}\right)\right)$, and platelet indices (MPV $(\mathrm{FL}), \mathrm{PCT}(\mathrm{L} / \mathrm{L})$, and PDW (\%) investigated for males and females are presented in Table 4 . A highly positive significant $(\mathrm{p} \leq 0.01)$ correlation was found between PLT and PCT in males, females, and all the dogs. In addition, there was a highly positive significant $(p \leq 0.01)$ correlation between MPV and PDW in all the dogs, while there was a low positive significant $(\mathrm{p} \leq 0.05)$ correlation in males. A highly positive significant $(p \leq 0.01)$ correlation was found between MPV and PCT in all the dogs, while a low positive significant $(\mathrm{p} \leq 0.05)$ correlation was found in males and females. A low positive significant $(\mathrm{p} \leq 0.05)$ correlation was found between PCT and PDW in all the dogs. There was no significant correlation between PLT and MPV, PDW in all the dogs.

The correlation between platelet indices and erythrocyte parameters $\mathrm{RBC}\left(\mathrm{x} 10^{12} / \mathrm{L}\right), \mathrm{MCV}(\mathrm{FL}), \mathrm{HCT}(\mathrm{L} / \mathrm{L})$, and RDW-CV (\%), investigated for males, females, and dogs are presented in table 5 . A highly negative significant $(\mathrm{p} \leq 0.01)$ correlation was found between PDW and RBCs and RDW $(\mathrm{CV})$ in males and all the dogs. In addition, there was a highly positive significant $(\mathrm{p} \leq 0.01)$ correlation between PDW and MCV in all the dogs. There was a low positive significant $(\mathrm{p} \leq 0.05)$ correlation in males. A highly negative significant $(\mathrm{p} \leq 0.01)$ correlation was found between MPV and RBCs in males and a low positive significant $(\mathrm{p} \leq 0.05)$ correlation in females. A highly negative significant $(\mathrm{p} \leq 0.01)$ correlation was found between MPV and RDW $(\mathrm{CV})$ in males. However, a highly positive significant $(p \leq 0.01)$ correlation was found between MPV and HCT in females. 
Table 1: Platelet count, indices, and erythrocyte parameters values in police dogs in Khartoum, Sudan

\begin{tabular}{lccc}
\hline Parameters $(\mathbf{n}=\mathbf{4 6})$ & Mean \pm SD & \multicolumn{2}{c}{$\mathbf{9 5 \%}$ Confidence Interval for Mean } \\
\cline { 3 - 4 } & & Upper Bound & Lower Bound \\
\hline PLT(x10 $/ \mathbf{L})$ & $172.02 \pm 52.72$ & 187.68 & 156.37 \\
MPV(FL) & $10.2 \pm 0.76$ & 10.43 & 9.98 \\
PCT(L/L) & $0.17 \pm 0.06$ & 0.190 & 0.16 \\
PDW (\%) & $11.16 \pm 0.31$ & 11.26 & 11.07 \\
RBCs (x10 & (12) & 6.74 & 6.56 \\
HGB(g/L) & $6.65 \pm 0.29$ & 180.52 & 173.78 \\
MCV(FL) & $177.15 \pm 11.35$ & 72.89 & 71.37 \\
HCT(L/L) & $72.13 \pm 2.57$ & 0.49 & 0.47 \\
MCH(Pg) & $0.48 \pm 0.02$ & 27.02 & 26.24 \\
MCHC(g/L) & $26.63 \pm 1.31$ & 375.9 & 363.05 \\
RDW-CV (\%) & $369.48 \pm 21.64$ & 17.85 & 7.63 \\
\hline
\end{tabular}

Table 2. Platelet count, indices, and erythrocyte parameters mean values in Labrador Retriever and German Shepherd dogs in Khartoum, Sudan

\begin{tabular}{lccc}
\hline Parameters (n=46) & $\begin{array}{c}\text { Labrador Retriever } \\
\text { mean } \pm \text { SD }\end{array}$ & $\begin{array}{c}\text { German Shepherd } \\
\text { mean } \pm \text { SD }\end{array}$ & P-value \\
\hline PLT(x10 $/ \mathbf{L})$ & $171.3 \pm 63.07$ & $172.58 \pm 44.49$ & 0.936 \\
MPV(FL) & $9.9 \pm 0.73^{\mathrm{b}}$ & $10.43 \pm 0.71^{\mathrm{a}^{*}}$ & 0.017 \\
PCT(L/L) & $0.16 \pm 0.06$ & $0.18 \pm 0.05$ & 0.131 \\
PDW (\%) & $11.25 \pm 0.38$ & $11.10 \pm 0.24$ & 0.136 \\
RBCs (x10 & $\mathbf{1 2} / \mathbf{L})$ & $6.75 \pm 0.26^{\mathrm{a}^{*}}$ & 0.004 \\
HGB(g/L) & $6.51 \pm 0.27^{\mathrm{b}}$ & $180.81 \pm 9.53^{\mathrm{a}^{*}}$ & 0.011 \\
MCV(FL) & $172.4 \pm 11.98^{\mathrm{b}}$ & $71.81 \pm 2.82$ & 0.337 \\
HCT(L/L) & $72.55 \pm 2.21$ & $0.48 \pm 0.03$ & 0.141 \\
MCH(Pg) & $0.47 \pm 0.021$ & $26.77 \pm 1.39$ & 0.411 \\
MCHC(g/L) & $26.45 \pm 1.21$ & $373.27 \pm 20.70$ & 0.178 \\
RDW-CV (\%) & $364.55 \pm 22.36$ & $17.84 \pm 0.37$ & 0.051 \\
\hline
\end{tabular}

$\mathrm{a}, \mathrm{b}$ means with superscript are significantly different at $\mathrm{p}<0.05$

Table 3: Platelet count, indices and erythrocyte parameters mean values in police dogs in male and female dogs in Khartoum, Sudan

\begin{tabular}{lccc}
\hline Parameters $(\mathbf{n}=\mathbf{4 6})$ & $\begin{array}{c}\text { Female } \\
\text { mean } \pm \text { SD }\end{array}$ & $\begin{array}{c}\text { Male } \\
\text { mean } \pm \text { SD }\end{array}$ & P-value \\
\hline PLT(x10/L) & $168 \pm 57.03$ & $176.81 \pm 48.03$ & 0.578 \\
MPV(FL) & $10.16 \pm 0.85$ & $10.24 \pm 0.65$ & 0.729 \\
PCT(L/L) & $0.17 \pm 0.06$ & $0.18 \pm 0.06$ & 0.34 \\
PDW (\%) & $11.08 \pm 0.22$ & $11.26 \pm 0.37$ & 0.056 \\
RBCs (x10 $/ \mathbf{1 2} / \mathbf{L})$ & $6.71 \pm 0.28$ & $6.57 \pm 0.28$ & 0.12 \\
HGB(g/L) & $178.64 \pm 11.83$ & $175.38 \pm 10.76$ & 0.338 \\
MCV(FL) & $71.72 \pm 2.09$ & $72.62 \pm 3.03$ & 0.242 \\
HCT(L/L) & $0.48 \pm 0.027$ & $0.48 \pm 0.02$ & 0.396 \\
MCH(Pg) & $26.60 \pm 1.18$ & $26.67 \pm 1.48$ & 0.858 \\
MCHC(g/L) & $370.68 \pm 19.45$ & $368.05 \pm 24.40$ & 0.686 \\
RDW-CV (\%) & $17.78 \pm 0.39$ & $17.70 \pm 0.39$ & 0.485 \\
\hline
\end{tabular}

\section{DISCUSSION}

The present study showed a difference in some hematological values (PLT, MCH, and $\mathrm{MCHC}$ ) which were higher when compared with the reference values reported by Weiss et al. (2011). MPV mean value was significantly (p $\leq 0.05$ ) higher in German Shepherd dogs compared with the Labrador Retriever dogs. Schneider and Mischke (2016) found that no significant difference in MPV values between
German Shepherd and Labrador Retriever dogs. This might be explained by the fact that MPV depends on PLT (number and size) and PCT, and it is calculated by dividing the PCT by the total number of PLT. In this study, PLT and PCT values were higher in German Shepherd dogs compared with the Labrador Retriever dogs this wasn't the case in Schneider and Mischke (2016) study. However, the high value of PCT indicates the presence of large Platelet in the 
bloodstream, which comes from immature platelets released from bone marrow due to the physiological response to exercise and stress.

RBCs mean value was significantly $(\mathrm{p} \leq 0.05)$ higher in German Shepherd compared with the Labrador Retriever. Çayir and Kozat (2016) found no significant difference in RBCs' mean value between German Shepherd and Labrador Retriever dogs. HGB mean value was significantly $(\mathrm{p} \leq 0.05)$ higher in German Shepherd compared with the Labrador Retriever dogs, this is in agreement with the study reported by Suljević et al. (2016), and in disagreement with Çayir and Kozat (2016) which found no significant difference in HGB mean value between German Shepherd and Labrador Retriever dogs. The difference between these studies results may come from and suggest that German Shepherd dogs have acclimatized to the dry tropical environment which is reflected in the similar RBC and HGB values more than Labrador Retriever dogs when both were raised under the tropical climate.

HCT mean value doesn't show any significance difference between two breeds, this is in line with (Çayir and Kozat 2016) and in contrast with (Suljević et al., 2016), which found a significant difference between two breeds. This might be explained by the fact that HCT depends on RBCs (number and size) and the body water. The authors found that RBCs values effected by both breed and environmental conditions and in general in dry tropical environments like in Sudan the animals have a low body water compare with cold climates.

Table 4: Correlation between Platelet Counts and Platelets Indices in Police Dogs

\begin{tabular}{|c|c|c|c|c|}
\hline Parameters & PLT $\left(\times 10^{9} / \mathrm{L}\right)$ & MPV (FL) & PCT (L/L) & PDW (\%) \\
\hline \multicolumn{5}{|c|}{ All Dogs } \\
\hline PLT(x10 $/$ L) & 1 & 0.290 & $0.874 * *$ & 0.289 \\
\hline MPV(FL) & 0.290 & 1 & $0.492 * *$ & $0.405 * *$ \\
\hline $\operatorname{PCT}(\mathrm{L} / \mathrm{L})$ & $0.874 * *$ & $0.492 * *$ & 1 & $0.343^{*}$ \\
\hline PDW (\%) & 0.289 & $0.405 * *$ & $0.343 *$ & 1 \\
\hline \multicolumn{5}{|c|}{ Males } \\
\hline $\operatorname{PLT}\left(\times 10^{9} / \mathrm{L}\right)$ & 1 & 0.343 & $0.980 * *$ & 0.164 \\
\hline $\operatorname{MPV}(\mathbf{F L})$ & 0.343 & 1 & $0.439 *$ & $0.536^{*}$ \\
\hline $\operatorname{PCT}(\mathrm{L} / \mathrm{L})$ & $0.980 * *$ & $0.439 *$ & 1 & 0.205 \\
\hline PDW (\%) & 0.164 & $0.536^{*}$ & 0.205 & 1 \\
\hline \multicolumn{5}{|c|}{ Females } \\
\hline PLT(x10 $/$ L) & 1 & 0.213 & $0.795 * *$ & 0.312 \\
\hline $\operatorname{MPV}(F L)$ & 0.213 & 1 & $0.492 *$ & 0.265 \\
\hline $\operatorname{PCT}(\mathrm{L} / \mathrm{L})$ & $0.795 * *$ & $0.492 *$ & 1 & 0.347 \\
\hline PDW (\%) & 0.312 & 0.265 & 0.347 & 1 \\
\hline
\end{tabular}

*Correlation significant at the $(\mathrm{p} \leq 0.05) ;{ }^{* *}$ Correlation significant at the $(\mathrm{p} \leq 0.01)$

Table 5: Correlation between Platelet Indices and Erythrocyte Parameters in Police Dogs

\begin{tabular}{|c|c|c|c|c|}
\hline Parameters & $\operatorname{PLT}\left(\times 10^{9} / \mathrm{L}\right)$ & $\operatorname{MPV}(F L)$ & $\operatorname{PCT}(\mathrm{L} / \mathrm{L})$ & PDW (\%) \\
\hline \multicolumn{5}{|c|}{ All Dogs } \\
\hline RBCs $\left(\times 10^{12} / \mathrm{L}\right)$ & -0.247 & 0.020 & -0.134 & $-0.381 * *$ \\
\hline MCV(FL) & 0.179 & $0.367 *$ & 0.201 & $0.420 * *$ \\
\hline $\operatorname{HCT}(L / L)$ & -0.126 & 0.240 & -0.019 & -0.075 \\
\hline RDW-CV (\%) & -0.271 & -0.230 & -0.251 & $-0.480 * *$ \\
\hline \multicolumn{5}{|c|}{ Males } \\
\hline $\operatorname{RBCs}\left(\times 10^{12} / \mathrm{L}\right)$ & -0.095 & $-0.552 * *$ & -0.090 & $-0.668 * *$ \\
\hline $\operatorname{MCV}(\mathbf{F L})$ & 0.185 & 0.363 & 0.211 & $0.471 *$ \\
\hline $\operatorname{HCT}(\mathrm{L} / \mathrm{L})$ & 0.105 & -0.180 & 0.132 & -0.184 \\
\hline RDW-CV (\%) & -0.042 & $-0.590 * *$ & -0.064 & $-0.637 * *$ \\
\hline \multicolumn{5}{|c|}{ Females } \\
\hline $\operatorname{RBCs}\left(\times 10^{12} / \mathrm{L}\right)$ & -0.341 & $0.445^{*}$ & -0.090 & -0.151 \\
\hline $\operatorname{MCV}(\mathrm{FL})$ & 0.112 & 0.323 & 0.158 & 0.291 \\
\hline $\operatorname{HCT}(L / L)$ & -0.241 & $0.511 * *$ & 0.005 & 0.053 \\
\hline RDW-CV (\%) & $-0.401 *$ & 0.050 & -0.322 & -0.352 \\
\hline
\end{tabular}

Adekola et al. (2015) found a significant difference in the erythrocyte parameters when compared across the different breeds (Alsatian, Rottweiler, Boerboel, Local, and others) even when they were raised in the same environment; this difference between breeds is in line with the current study (Breed can have effect on hematological parameters).This 
difference between breeds can be referred to genetic predisposition and other exogenous and endogenous factors, such as the type of police work which can affect platelets indices and erythrocyte parameters in Sudan the German Shepherd police dogs work on the tasks that need great physical exertion, such as chasing criminals and resolving the riots. This requires extensive physical training; while Labrador Retriever dogs work in missions that require less effort, such as detecting drugs and explosives, these tasks require less physical effort and less physical training (Abdeen, 2005). Training for long-duration need more oxygen and more blood for hemostasis that activate the bone marrow to produce more blood especially (RBCs and Platelets) (Weiss et al.,2011; Suljević et al., 2016).

No significant difference between males and females in MPV and PDW, this is in line with Awad-Elkareem et al (2015) in humans, Schneider and Mischke ((2016), and Nidaa et al. (2017) while contradicting with the finding of Hussein et al. (2012) in camels.

In the current study, there was no significant difference between males and females in PLT which is in agreement to Hussein et al (2010; 2012) in camels, Adekola et al. (2015), Awad-Elkareem et al. (2015) in human and Schneider and Mischke (2016) in dogs. However, this result is in contrast with the finding of Nidaa et al (2017) in dogs., which may be as a result of breed difference, method of sampling and the device used in the examination of the samples.

Furthermore, platelet count and indices mean values were higher in males compared with females. This can be attributed to hormonal profiles amongst the gender (Nagata et al., 2003). The process by which megakaryocytes proceed to proplatelet formation and platelet production is reported to increase under influence of autocrine estrogen, and less so under the influence of Progesterone hormone (Nagata et al., 2003). As a result, that the physiological status (estrus, pregnancy, and lactation) can affect platelet indices. Also, the number of members of any breed in any group (Females and Males) can affect it.

On other hand, erythrocyte parameters (RBCs, HGB, HCT, $\mathrm{MCHC}$, and $\mathrm{RDW}-\mathrm{CV}$ ) values were higher in females compared with the males. This can be attributed to hormonal profiles amongst the gender. Male androgenic hormone, testosterone, has been reported to stimulate the production of erythropoietin, which in turn increases the process of erythropoiesis and consequently, leads to higher erythrocyte values in males' animals (Erickson et al., 2015). These male hormones increase significantly in the mating season (Erickson et al., 2015) and therefore their effect appears clearly in the process of erythropoiesis.

As this study was conducted outside the mating season, where the concentration of male hormones is low it is therefore worthy to note that, physiological status (estrus, pregnancy, and lactation) can affect erythrocyte parameters (Ajala et al., 2011). In this study, the females may have some physiological conditions like early pregnancy which cannot be detected and have a positive impact on hematological parameters. The number of members of any breed in any group (Females and Males) can affect the erythrocyte parameter (Bourgès-Abella et al., 2011; Suljević et al., 2016; Aniołek et al., 2018).

The present study revealed a positive significant correlation between PLT and PCT in males, females, and all the dogs, this is in line with the finding of Wiwanitkit (2004) in human, Hussein et al. (2009) in Arabian mountain gazelle (Hussein et al., 2010; Hussein et al.,2012) in camels. Nidaa et al. (2017) found a similar result in German Shepherd dogs except that males showed a low positive significant correlation between the two parameters. The highly positive significant correlation between MPV and PDW in all the dogs and the low positive significant correlation in males are in line with the finding of Hussein et al. (2012) in camels.

Hussein et al. (2010) found a similar result in camels except that all the camels showed a low positive significant correlation between the two parameters. Wiwanitkit (2004) in humans, Hussein et al. (2009) in Arabian mountain gazelle, and Nidaa et al (2017) in German shepherd dogs found different results.

A highly positive significant correlation was found between MPV and PCT in all the dogs, low positive significant correlation was found in males and females. This is in line with Hussein et al. (2012) in camels and in contrast with the finding of Wiwanitkit (2004) in human, Hussein et al. (2009) in Arabian mountain gazelle, Hussein et al. (2010) in camels, and Nidaa et al, (2017) in German Shepherd dogs. No significant correlation was found between PLT and MPV, PDW in all the dogs and this agrees with Wiwanitkit (2004) in human, Hussein et al. (2009) in Arabian mountain gazelle, Hussein et al. (2010) in camels, and Nidaa et al. (2017) in German Shepherd dogs, and is in contrast with the finding of Hussein et al. (2012) in camels.

In the current study, there was a highly negative significant correlation between PDW and RBCs in males and all the dogs. This is in line with Nidaa et al (2017) in German Shepherd dogs except that males did not show any significant correlation between the two parameters. This is in contrast with the finding of Hussein et al. (2009) in Arabian mountain gazelle, Hussein et al. (2010), and Hussein et al. (2012) in camels. High negative significant correlations were found between PDW -and RDW (CV) in males and all the dogs. Hussein et al. (2010) and Hussein et al. (2012) found a similar result in all the camels.

Nidaa et al. (2017) found similar in German Shepherd dogs except that males did not show any significant correlation between the two parameters. Wiwanitkit (2004) in humans and Hussein et al. (2009) in Arabian mountain gazelle found dissimilar results.

A highly positive significant correlation was found between PDW and MCV in all the dogs and a low positive significant correlation in males. This is in line with Wiwanitkit (2004) in human, Hussein et al. (2012) in camels, Nidaa et al. (2017) in German Shepherd dogs except that females showed a significant correlation between the two parameters, and in contrast with the finding 
of Hussein et al. (2009) in Arabian mountain gazelle and Hussein et al. (2010) in camels.

A highly negative significant correlation was found between MPV and RDW (CV) in males. Hussein et al (2012) in camels and Nidaa et al (2017) in German Shepherd dogs found a low significant correlation between the two parameters. Wiwanitkit, (2004) in humans, Hussein et al (2009) in Arabian mountain gazelle, and Hussein et al (2010) in camels, found similar results. However, a highly positive significant correlation between MPV and HCT in females and a highly negative significant correlation between MPV and RBCs in males were found, this is in contrast with the finding of previous studies in human, Arabian mountain gazelle, camels, and dogs.

\section{Conclusion}

The present results concluded that the result was within the international normal range but slightly different from the previous study done in Sudan in German shepherd dogs. $\mathrm{MPV}, \mathrm{RBCs}, \mathrm{HGB}$, and RDW-(CV) mean values were different in the two breeds studied. This study revealed a significant correlation between platelet count and platelet indices and between platelet indices and erythrocyte parameters. This correlation should take into consideration in the prognosis, diagnosis and clinical interpretations of blood diseases especially those related to platelets.

\section{Conflict of Interest}

The authors have no conflict of interest to declare.

\section{Authors Contribution}

AAA and SAO designed the research and supervised the project. AAA was involved in blood sample collection and parameter evaluation. MA participated in the statistical analysis, literature and manuscript review. All authors have read and approved the final manuscript.

\section{REFERENCES}

Abdeen, A. M. (2005). Effects of Smelling Narcotics on Some Biochemical Parameters in German Shepherd Dogs. M.Sc. thesis, University of Khartoum, Sudan.

Adebiyi, O. E., Ajayi, J. O. and Olayemi, F. O. (2014). Haematology of Rottweiler dog in a tropical environment. New York Science Journal, 7(9): 1-4.

Adekola, A. A., Jagun, A. T., Emikpe, B. O. and Antia, R. E. (2015). Baseline haematology and erythrocyte morphological changes of apparently normal dogs raised in Ibadan, Oyo State. Nigerian Journal of Physiological Sciences, 30(1-2) 111-118.

Ajala, O. O., Fayemi, O. O., and Kolawole, T. O. (2011). Short Communication Erythrocyte parameters as indicators for differentiating between the pregnant and pseudo pregnant bitches in Nigeria. Nigerian Journal of Physiological Sciences, 26(1) $19-22$.

Aniołek, O., Barc, A., Jarosińska, A., and Gajewski, Z. (2018). Evaluation of frequency and intensity of asymptomatic anisocytosis in the Japanese dog breeds Shiba, Akita, and Hokkaido. Acta Veterinaria Brno, 86(4), 385-391.

Awad-Elkareem, A., Israa, I., Razaz, Y., Eman, A Reem, M., Sara, M., Haifa, A. and Adam., A. (2015). Reference value of Platelets count and indices in Sudanese using Sysmex KX-21. International Journal of Healthcare Sciences, 3(2): 120-125.

Bommer, N. X., Shaw, D. J., Milne, E. M., and Ridyard, A. E. (2008). Platelet distribution width and mean platelet volume in the interpretation of thrombocytopenia in dogs. Journal of Small Animal Practice, 49(10), 518-524.

Bourgès-Abella, N., Geffré, A., Concordet, D., Braun, J. P., and Trumel, C. (2011). Canine reference intervals for the Sysmex XT-2000iV hematology analyzer. Veterinary Clinical Pathology, 40(3), 303-315.

Çayir, C. and Kozat, S. (2016). Investigation of homocysteine levels in healthy dogs Journal of Veterinary Science and Animal Husbandry, 4(3): 305-310.

Erickson, H. H., Goff, J. P., and Uemura, E. E. (2015). Dukes' physiology of domestic animals. A John Wiley \& Sons, Ltd.Chichester, UK.

Fabisiak, M., Sapierzynski, R. and Klucinski, W. (2010). Analysis of haematological abnormalities observed in dogs infected by a large Babesia. Bulletin of the Veterinary Institute in Pulawy, 54: 167-170.

Hussein, M. F., Al-Jumaah, R. S., Alhaidary, A. A., Alshaikh, M. A., Elnabi, A. G., Mohammed, O. B. and Macasero, W. V. (2009). Blood Platelet Indices and Parallel Red Cell Parameters in the Arabian Mountain Gazelle (Gazella gazella). Research Journal of Biological Sciences, 4(7): 785788.

Hussein. M., Aljumaah. R., Alhaidary. A., Alshaikh. M., Mohammed. O., Omer. S., Gar-Elnabi. A. and Macasero. W. (2010). Platelets indices of the dromedary camel (Camelus dromedaries). Veterinarski Arhiv, 80 (3):375-382.

Hussein, M. F., Elnabi, A. G., Aljarf, A. N., Aljumaah, R. S., Bakhiet, A. O. and Alshaikh, M. A. (2012). Variation of the platelet indices of dromedary camel (Camelus dromedarius) with age, sex and breed. African Journal of Biotechnology, 11(19): 4478-4483.

Nagata, Y., Yoshikawa, J., Hashimoto, A., Yamamoto, M., Payne, A. H. and Todokoro, K. (2003). Proplatelet formation of megakaryocytes is triggered by autocrine-synthesized estradiol. Genes \& Development, 17(23):2864-2869.

Nidaa, A.M., Ahmed. S.H, Shadia, A., O. and Abdelsammad, G., H. (2017) "Platelet Indices in Male and Female German Shepherd Dogs in the Sudan." IOSR Journal of Agriculture and Veterinary Science, 10(7): 60-63.

Reddy, R. S., Khan, I. M. and Phansalkar, D. M. (2015). Platelet Distribution Width (PDW) in Thrombocytopenia. Indian Medical Gazette, 188: 169-174. 
Santimone, I., Di Castelnuovo, A. F., De Curtis, A., Spinelli, M., Cugino, D., Gianfagna, F. and Iacoviello, L. (2011). White blood cells count, sex and age are major determinants of platelet indices heterogeneity in an adult general population: results from the MOLI-SANI project. Haematologica, 96.8, 1180-1188.

Schneider, L. and Mischke, R. (2016). Platelet variables in healthy dogs: reference intervals and influence of age, breed and sex. Comparative Clinical Pathology, 25(6):1097-1106.

Schwartz, D., Sharkey, L., Armstrong, P. J., Knudson, C. and Kelley, J. (2014). Platelet volume and plateletcrit in dogs with presumed primary immune-mediated thrombocytopenia. Journal of Veterinary Internal Medicine, 28(5):1575-1579.

Suljević, D., Muhamed, F., Dunja, R. and Amir, Z. (2016). Comparative analysis of hematological parameters in working police dogs. Macedonian Journal of Animal Science, 6(1):65-70.
Temizel, E.M., Cihan, H., Yilmaz, Z. and Aytug, N. (2011). Evaluation of erythrocyte and platelet indices in canine visceral leishmaniasis. Ankara Üniversitesi Veteriner Fakültesi Dergisi, 58(3):185-188.

Vojta, L., Mrljak, V. and Beck, R. (2012). Haematological and biochemical parameters of canine hepatozoonosis in Croatia. Veterinarski Arhiv, 82(4):359-370.

Weiss, D.J. and Wardrop, K.J. E. (2011). Schalm's veterinary hematology. A John Wiley \& Sons, Ltd., Publication. Chichester, UK.

Wiwanitkit, V. (2004). Plateletcrit, mean platelet volume, platelet distribution width: its expected values and correlation with parallel red blood cell parameters. Clinical and Applied Thrombosis/Hemostasis, 10(2), 175-178.

Żmigrodzka, M., Guzera, M. and Winnicka, A. (2014). Evaluation of reticulated platelets in dogs witbreed-related thrombocytopenia. Polish Journal of Veterinary Sciences, 17(1):137-142. 